\title{
CARACTERIZACIÓN DE UN BOSQUE DE QUEÑUAL (Polylepis spp.) UBICADO EN EL DISTRITO DE HUASTA, PROVINCIA DE BOLOGNESI (ANCASH, PERÚ)
}

\section{CHARACTERIZATION OF A QUEÑUAL (Polylepis spp.) FOREST AT HUASTA DISTRICT, BOLOGNESI PROVINCE (ANCASH, PERU)}

\author{
Andrés Castro ${ }^{1}$ y Mercedes Flores ${ }^{2}$
}

\begin{abstract}
Resumen
Los bosques del género Polylepis, a pesar de cumplir un rol central en la ecología de los Altos Andes, constituyen en la actualidad uno de los ecosistemas más vulnerables de Sudamérica. Sin embargo, la percepción de su valor está cambiando y se está reduciendo su deforestación e incentivando su conservación. Con el objetivo de caracterizar la población de dos especies de Polylepis ( $P$. reticulata y $P$. sericea) presentes en el bosque de la microcuenca de Paria - distrito de Huasta, se muestreó 109 parcelas de $400 \mathrm{~m}^{2}$, donde se evaluaron los árboles con DAP mayor o igual a $3 \mathrm{~cm}$ en sub-parcelas de $200 \mathrm{~m}^{2}$ y a la regeneración natural en sub-parcelas de $25 \mathrm{~m}^{2}$. Este bosque se caracteriza porque la mayoría de los renuevos de $P$. reticulata tienen alturas entre $8-33$ $\mathrm{cm}$, cobertura vegetal entre $1-12 \%$ y densidades entre $11-42$ individuos/sub-parcela; el DAP de los árboles por lo general varía entre $9-26 \mathrm{~cm}$, alturas entre $5-11 \mathrm{~m}$ y densidades entre $6-17$ individuos/sub-parcela. La mayoría de los renuevos de $P$. sericea se caracterizan por presentar alturas entre $25-63 \mathrm{~cm}$; los árboles presentan por lo general DAP entre $11-20 \mathrm{~cm}$ y alturas entre $4-7 \mathrm{~m}$. En conclusión, se sugiere que el bosque de Polylepis de la microcuenca de Paria - distrito de Huasta sería uno de los más antiguos y mejor conservados del norte del Perú.
\end{abstract}

Palabras clave: Queñua, bosques andinos, Polylepis reticulata, Polylepis sericea, regeneración, morfometría, Huasta.

\begin{abstract}
Although Polylepis forests play a major role in high Andean ecology, they are one of the most vulnerable ecosystems in South America. However the perception of their value is changing, reducing its deforestation and encouraging its conservation. The goal of this research was to characterize the populations of two Polylepis species ( $P$. reticulata and P. sericea) located in the Paria micro-basin, Huasta District. A number of 109 patches were evaluated. $200 \mathrm{~m} 2$ sub-patches inside $400 \mathrm{~m}^{2}$ sized patches were used to assess trees with higher than $3 \mathrm{~cm} \mathrm{DBH}$, and natural regeneration was assessed in $25 \mathrm{~m}^{2}$ sub-patches. For $P$. reticulate trees, renewals have heights usually ranging from 8 to $33 \mathrm{~cm}$, plant coverage varies between $1-12 \%$, and a population density of $11-42$ individuals/sub-patch is found; DBH generally varies between $9-26 \mathrm{~cm}$; the height ranges from 5 to $11 \mathrm{~m}$, and population densities vary between $6-17$ individuals/sub-patch. For $P$. sericea, the majority of renewals are $25-63 \mathrm{~m}$ high; the trees have DBH from 11 to $20 \mathrm{~cm}$ and are $4-7 \mathrm{~m}$ high. We suggest that the Polylepis forest in the Paria micro-basin, Huasta District, would be one of the oldest and best preserved in Northern Peru.

Key words: Queñua, Andean forests, Polylepis reticulata, Polylepis sericea, regeneration, morphometry, Huasta.
\end{abstract}

\section{Introducción.}

En la actualidad por encima de los $3500 \mathrm{~m}$, la vegetación de los Andes centrales se encuentra dominada por zonas agrícolas, pastizales y zonas arbustivas (Kessler, 2006). Los bosques naturales son bastante difíciles de ver, generalmente se encuentran relegados a laderas rocosas o quebradas (Kessler, 2006; León, 2009; Mendoza \& Cano, 2012). Las especies leñosas presentes en estos bosques pertenecen a los géneros: Buddleja, Clethra, Gynoxys,
Podocarpus o Prumnopitys, pero las especies arbóreas dominantes en estos bosques son las del género Polylepis (Kessler, 2006).

Polylepis incluye aproximadamente de 15 a 27 especies entre árboles y arbustos, según diferentes autores (Simpson, 1979; Kessler \& Schmidt-Lebuhn, 2006; Kessler, 2006; Mendoza \& Cano, 2012). Dentro de la totalidad de especies, la gran mayoría son árboles de $5-10 \mathrm{~m}$ de altura, algunas son arbustivas (P. microphylla, $P$. pepei, $P$. tarapacana, $P$. 
Enero - Julio 2015

tomentella subsp. nana) y unas cuantas pueden llegar a superar los $25 \mathrm{~m}$ de altura ( $P$. lanata, $P$. pauta) (Kessler, 2006). El género Polylepis únicamente se distribuye a lo largo de los Andes tropicales y subtropicales de Sudamérica, abarcando desde Venezuela hasta el norte de Argentina y Chile (Simpson, 1979; Arévalo \& Recharte, 2003). En el Perú se reportan 19 especies (5 endémicas), siendo de esta manera el país que presenta la mayor diversidad en el género (Simpson, 1979; Kessler \& Schmidt-Lebuhn, 2006; Mendoza \& Cano, 2011). El nombre común usado por los comuneros del distrito de Huasta es "Quenual".

Los bosques de Polylepis representan uno de los ecosistemas más vulnerables de los altos Andes, por la creciente presión humana debido a factores económicos, sociales y culturales (Servat et al., 2002). Sin embargo estos ecosistemas cumplen un rol central en la ecología altoandina, como hábitats de muchas especies de plantas y animales, como fuente importante de recursos para los habitantes locales, captador de $\mathrm{CO}_{2}$ atmosférico, formación de suelo, plantas medicinales asociadas y regulador del ciclo hídrico del agua (Fjeldså \& Kessler, 1996; Venero \& De Macedo, 1983; Arévalo \& Recharte, 2003; Kessler, 2006; León, 2009).

En la actualidad la microcuenca de Paria (de 767.34 ha), distrito de Huasta, es reconocida por el Ministerio del Ambiente como un Área de Conservación Privada (R.M. N 306-2011-MINAM), que busca conservar la diversidad biológica de la microcuenca, en especial, los bosques del género Polylepis (de 99 ha). El bosque de la microcuenca de Paria, motivo del presente estudio (de 23 ha) es el más alejado del pueblo (a $10.63 \mathrm{~km}$ de distancia), mejor

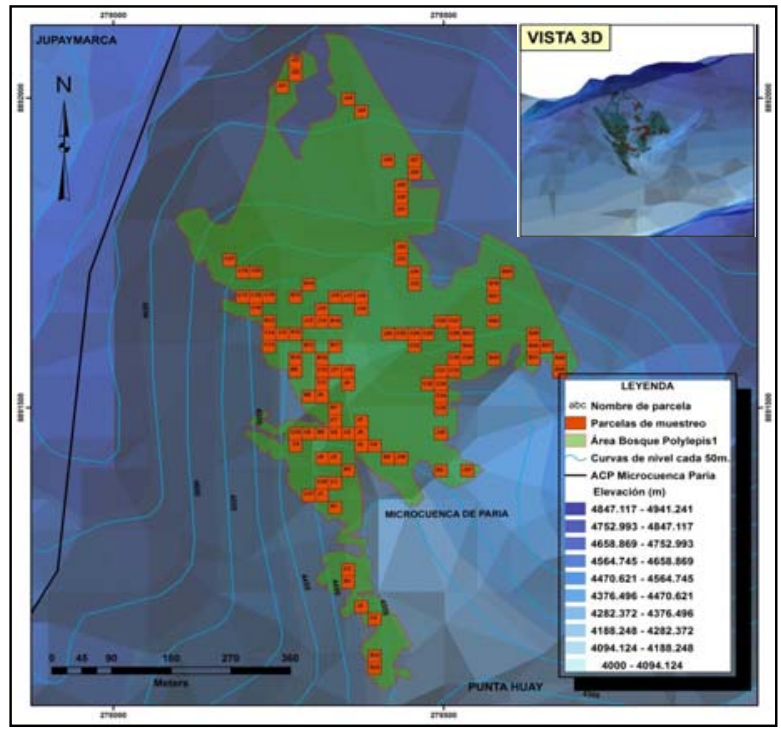

Figura 2. Localización de las unidades muestrales (escala 1:3000).

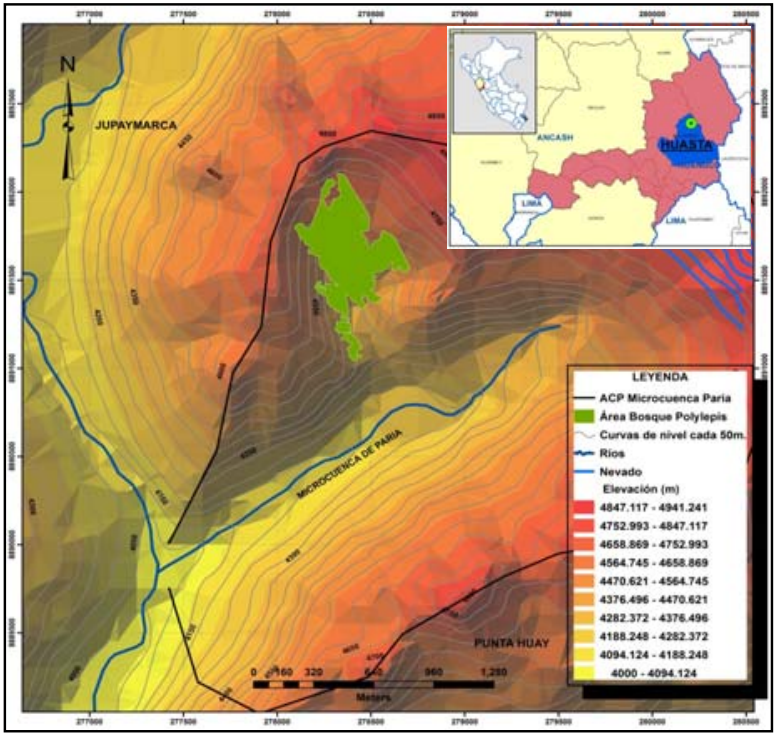

Figura 1. Localización del bosque de Polylepis en estudio (escala 1:10000).

conservado y el que posee los individuos de mayor envergadura; por lo que es considerado uno de los bosques más antiguos de la zona según los pobladores del distrito. En la actualidad, está destinado a prácticas de conservación y ecoturismo, por lo que es de suma importancia caracterizar el bosque para de esta manera fortalecer los conocimientos básicos, con la finalidad de conservarlo y ser punto de partida para futuras acciones aplicadas como: estudios de dendrocronología, diversidad de aves, dispersión de semillas, sistemas de polinización y otros.

En este estudio se caracterizan a los individuos de porte arbóreo y regeneración del género Polylepis, mediante la medición de la altura, cobertura, densidad y diámetro.

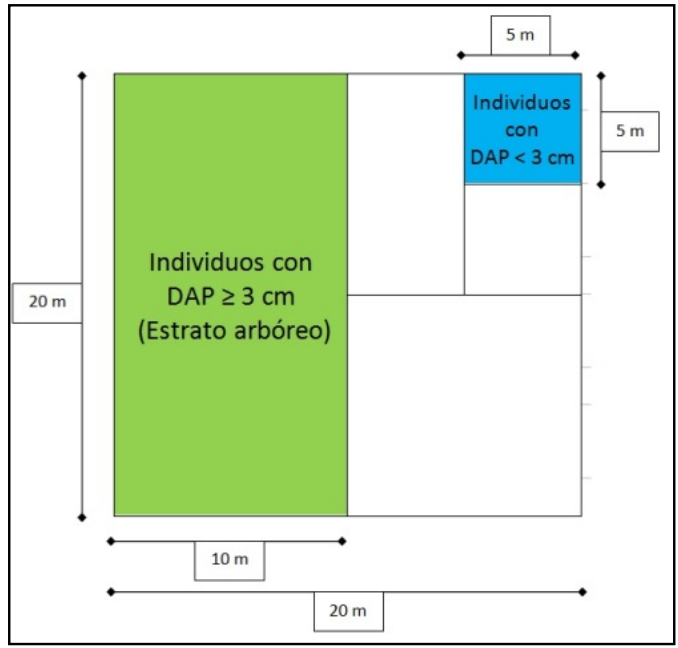

Figura 3. Diseño de muestreo utilizado en cada parcela de $400 \mathrm{~m}^{2}$. 
Materiales y métodos.

Descripción de la zona de estudio.

Este estudio se realizó en el bosque de Polylepis (4350 a $4700 \mathrm{msnm}$ ) ubicado en la microcuenca de Paria, perteneciente al distrito de Huasta, provincia de Bolognesi, departamento de Ancash - Perú (Figura 1). El bosque presenta los siguientes límites (CGS WGS84): Al norte, $10^{\circ} 1^{\prime} 0.08^{\prime}$ ' S / 77 1 '22.18' $\mathrm{O}$; al sur, $10^{\circ} 1{ }^{\prime} 33.05^{\prime}$ ' S / 77'1'18.18' O; al este,

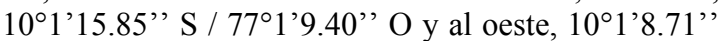
$\mathrm{S} / 7^{\circ} 1$ '22.99', O. El bosque se desarrolla en la ecorregión "Puna y los Altos Andes" (Brack et al., 2000) y se encuentra localizado entre el Parque Nacional Huascarán y la Zona Reservada Cordillera de Huayhuash, proporcionando conectividad ecológica entre los bosques remanentes del género Polylepis. Colecta y determinación de especies.

Se realizaron colectas botánicas durante la salida de campo en la estación seca (Setiembre 2010), ya que en esta estación se encontraron los individuos en su fase reproductiva (con flores y/o frutos). Las muestras fueron identificadas en el Herbario de San Marcos (USM) del museo de Historia Natural.

Diseño de muestreo.

El bosque tiene un área total de 23 ha, comprendiendo 575 parcelas de $400 \mathrm{~m}^{2}$, de las cuales se seleccionaron 109 parcelas (Figura 2) mediante un muestreo aleatorio simple. Cada parcela fue dividida en sub-parcelas con la finalidad de analizar por separado los renuevos de los árboles. Los renuevos se analizaron en sub-parcelas de 5 x $5 \mathrm{~m}^{2}$ y los árboles en sub-parcelas $20 \times 10 \mathrm{~m}^{2}$ (Figura 3). Se analizaron sólo árboles con diámetro mayor o igual a $3 \mathrm{~cm}$, debido a la no existencia de árboles con un diámetro menor. Evaluaciones realizadas.

A. Regeneración natural (analizados en sub-parcelas de 5 x $5 \mathrm{~m}^{2}$ ). Se caracterizó a los renuevos mediante la medición de las siguientes variables:

a.1Altura $(\mathrm{cm})$, fue medida desde la intersección de la base del tallo con el suelo hasta la rama más alta.

a.2Cobertura total (\%), fue medida utilizando el área de una elipse que se asemeja a la forma de la copa de Polylepis. Luego se determinó la superficie total cubierta por sub-parcela.

a.3Densidad (individuos/sub-parcela), número total de individuos vivos por sub-parcela.

B. Árboles (analizados en sub-parcelas de $20 \times 10$ $\mathrm{m}^{2}$ ).

b.1Altura $(\mathrm{m})$, fue medida mediante proyección visual. Para esta medición una persona se ubicó junto a un árbol sosteniendo una vara de $4 \mathrm{~m}$ de alto, y luego un anotador desde una zona más alta realizó la proyección con la vista y anotó la altura. Debido a que es un método de medición rápido pero no muy exacto, se trabajó con un error de estimación de $\pm 10 \%$.

b.2Diámetro a la altura del pecho $(\mathrm{cm})$, se aplicó la metodología de Rügnitz et al (2009), para esto el diámetro del tronco se midió a $1.30 \mathrm{~m}$ de la superficie del suelo.

b.3Densidad (individuos/sub-parcela), número total de individuos vivos por sub-parcela.

Análisis de datos.

Para el análisis de los datos se utilizó el software estadístico Minitab versión 16.1.0 (Minitab Inc., 2010). Los análisis estadísticos utilizados en el procesamiento de los datos fueron los siguientes:

-Estadísticas descriptivas.

-Agrupación de datos en intervalos de clase y uso de gráficos de distribución.

Gráfico de cajas.

\section{Resultados.}

Especies del género Polylepis que forman el bosque.

En el bosque de Polylepis hay dos especies: $P$. reticulata Hieron. y $P$. sericea Wedd. La especie predominante a lo largo del bosque fue $P$. reticulata (ocupa $18.5 \mathrm{ha}$ ); mientras que $P$. sericea ocupa tan sólo 4.5 ha, por lo que sólo se pudo muestrear en cinco parcelas (J21 a J25) ubicadas en la zona más alta del bosque (4659 a $4691 \mathrm{msnm}$ ). Además, se observó que las zonas colonizadas por los árboles de $P$. reticulata presentaban por lo general coberturas superiores al $80 \%$, contrastando con los árboles de $P$. sericea que se encontraban bastante dispersos $y$ presentaban coberturas por debajo del $25 \%$.

Tabla 1. Estadísticas descriptivas de las variables analizadas en el estudio.

\begin{tabular}{|c|c|c|c|c|c|c|c|c|c|}
\hline & \multicolumn{4}{|c|}{ Regeneración natural } & \multicolumn{5}{|c|}{ Porte arbóreo } \\
\hline & \multicolumn{2}{|c|}{$\begin{array}{l}\text { Altura } \\
(\mathrm{cm})\end{array}$} & \multirow{2}{*}{$\begin{array}{c}\text { Densidad } \\
\text { (ind/parcela) } \\
\text { Pr-H }\end{array}$} & \multirow{2}{*}{$\begin{array}{c}\text { Cobertura } \\
(\%) \\
\text { Pr-H }\end{array}$} & \multicolumn{2}{|c|}{$\begin{array}{l}\text { DAP } \\
(\mathrm{cm})\end{array}$} & \multicolumn{2}{|c|}{$\begin{array}{c}\text { Altura } \\
\text { (m) }\end{array}$} & \multirow{2}{*}{$\begin{array}{c}\text { Densidad } \\
\text { (ind/parcela) } \\
\text { Pr-H }\end{array}$} \\
\hline & $\operatorname{Pr}-\mathrm{H}$ & $P s-\mathrm{W}$ & & & Pr-H & Ps-W & Pr-H & Ps-W & \\
\hline Media & 31.05 & 48.46 & 21.53 & 9.54 & 19.97 & 16.92 & 8.67 & 5.35 & 12.00 \\
\hline Mínimo & 0.50 & 10.00 & 0.00 & 0.02 & 3.18 & 3.50 & 1.14 & 2.00 & 1.00 \\
\hline Q1 & 8.00 & 24.50 & 2.00 & 1.26 & 9.23 & 10.82 & 5.00 & 4.00 & 6.00 \\
\hline Mediana & 16.00 & 42.00 & 16.00 & 3.95 & 16.23 & 14.32 & 7.50 & 5.25 & 11.00 \\
\hline Q3 & 33.00 & 62.50 & 35.75 & 11.72 & 26.42 & 20.37 & 11.25 & 7.00 & 16.50 \\
\hline Máximo & 330.00 & 195.00 & 93.00 & 100.00 & 108.86 & 50.29 & 26.00 & 11.00 & 34.00 \\
\hline
\end{tabular}




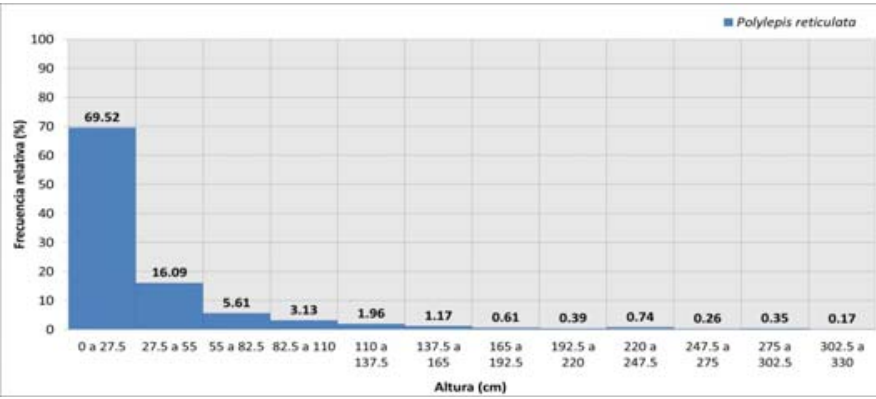

Figura 4. Distribución de alturas de renuevos de $P$. reticulata.

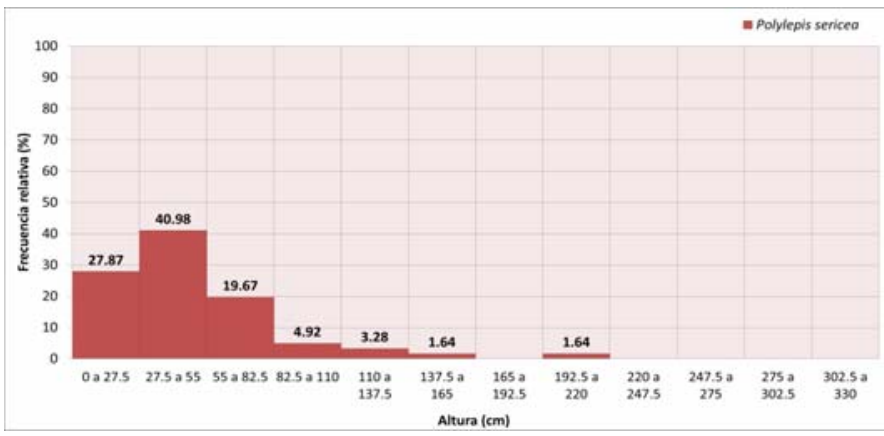

Figura 5. Distribución de alturas de los renuevos de P. sericea.

Análisis de la regeneración o renuevos.

Se muestrearon en total 2300 individuos de los cuáles 2239 eran $P$. reticulata y 61 eran $P$. sericea.

Análisis de la altura (cm).

La distribución de alturas de los individuos regenerados se puede observar en las Figuras 4 y 5 . La altura mínima para la especie $P$. reticulata fue de 0.5 cm y la máxima de $330 \mathrm{~cm}$ (Tabla 1), además el $50 \%$ de los individuos presentan alturas entre 8 a $33 \mathrm{~cm}$ (Figura 6, Tabla 1). Para $P$. sericea, la altura mínima fue de $10 \mathrm{~cm}$ y la máxima de $195 \mathrm{~cm}$ (Tabla 1); además el $50 \%$ de los individuos presentaron alturas entre 24.5 a $62.5 \mathrm{~cm}$ (Figura 7, Tabla 1).

Análisis de la densidad (indiv./sub-parcela).

Se presentaron individuos regenerados de $P$. reticulata en 84 sub-parcelas, representando el $82 \%$ del total. La distribución de las densidades obtenidas se pueden observar en la Figura 8. La sub-parcela $\mathbf{J} 4$ presentó la mayor densidad de individuos regenerados (93 individuos/sub-parcela) ubicada a $4410 \mathrm{msnm}$ (Figura 2, Tabla 1). Además el $50 \%$ de las subparcelas presentaron densidades entre 11 a 42 individuos/sub-parcela (Figura 9).

Cobertura vegetal por parcela (\%).

En la Figura 10 se muestra la distribución de las coberturas obtenidas en las 84 sub-parcelas de $P$. reticulata. La sub-parcela $\mathbf{J} 3$ presentó un $100 \%$ de cobertura (Figura 2, Tabla 1). Además el $50 \%$ de las sub-parcelas presentaron coberturas entre 1.2 a 11.7 $\%$ (Figura 11, Tabla 1).
Análisis de los individuos Arbóreos.

Se analizaron en total a 1301 individuos de los cuáles 1255 eran $P$. reticulata y 46 eran $P$. sericea. Para el caso de la variable altura se tomó en cuenta al $83 \%$ de los individuos, ya que no se incluyeron los individuos que estaban postrados $(17 \%)$.

Diámetro a la altura del pecho $(\mathrm{cm})$.

La distribución de diámetros de los individuos arbóreos se puede observar en las Figuras 12 y 13. El diámetro mínimo para la especie $P$. reticulata fue de $3.2 \mathrm{~cm}$ y el máximo de $108.9 \mathrm{~cm}$ (Tabla 1), además el $50 \%$ de los individuos presentaron diámetros entre 9.4 a $26.4 \mathrm{~cm}$ (Figura 14, Tabla 1). Con respecto a la especie $P$. sericea, el diámetro mínimo fue de $3.5 \mathrm{~cm}$ y el máximo de $50.3 \mathrm{~cm}$ (Tabla 1); además que el $50 \%$ de los individuos presentaron diámetros entre 10.8 a $20.4 \mathrm{~cm}$ (Figura 15, Tabla 1).

Altura $(m)$.

La distribución de alturas de los individuos arbóreos se puede observar en las Figuras 16 y 17. La altura mínima para la especie $P$. reticulata fue de $1.1 \mathrm{~m}$ y la máxima de $26 \mathrm{~m}$ aproximadamente (Tabla

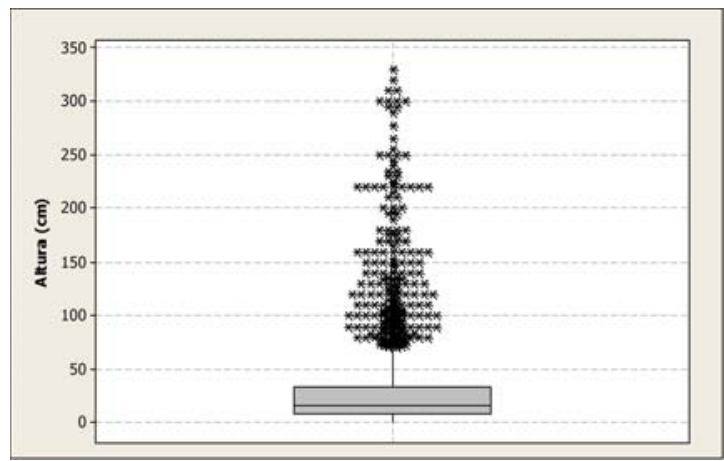

Figura 6. Distribución de alturas $(\mathrm{cm})$ de los renuevos de $P$. reticulata.

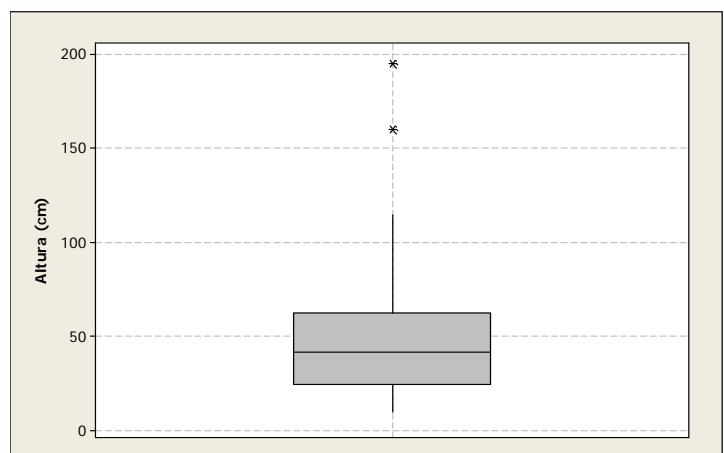

Figura 7. Distribución de alturas $(\mathrm{cm})$ de los renuevos de $P$. sericea. 


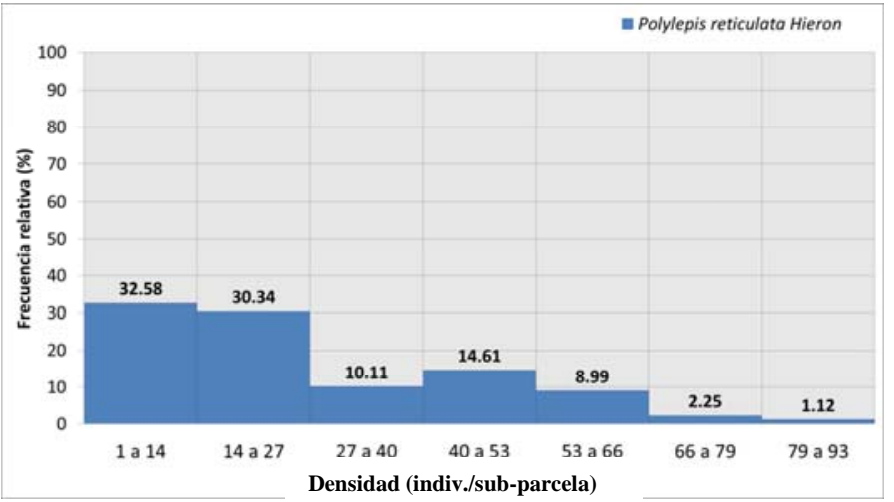

Figura 8. Distribución de densidades de los renuevos de $P$. reticulata en sub-parcelas de $25 \mathrm{~m}^{2}$.

1), además el $50 \%$ de los individuos presentaron alturas entre 5 a $11.3 \mathrm{~m}$ (Figura 18, Tabla 1). Con respecto a la especie $P$. sericea, la altura mínima fue de $2 \mathrm{~m}$ y la máxima de $11 \mathrm{~m}$ (Tabla 1); además el 50 $\%$ de los individuos presentaron alturas entre 4 a $7 \mathrm{~m}$ (Figura 19, Tabla 1).

Análisis de la densidad (indiv./sub-parcela).

La distribución de las densidades obtenidas se pueden observar en la Figura 20. La sub-parcela con mayor densidad de individuos de porte arbóreo fue la C20 (33 indiv./sub-parcela, $4534 \mathrm{msnm}$ ) y J16 (34 indiv./sub-parcela, $4503 \mathrm{msnm}$ ) (Figura 2, Tabla 1). Además el $50 \%$ de las sub-parcelas presentaron densidades entre 6 a 17 indiv./sub-parcela (Figura 21, Tabla 1).

\section{Discusión.}

La especie $P$. reticulata se encontró hasta una altitud máxima de $4640 \mathrm{msnm}$, contrastando de esta manera con el estudio de Mendoza \& Cano (2011), que reportan una altitud máxima de $4450 \mathrm{msnm}$. Lo mismo sucede con la especie $P$. sericea, ya que Mendoza \& Cano (2011) reportan una altitud máxima de $4100 \mathrm{msnm}$, contrastando con individuos que en el presente estudio fueron encontrados hasta los 4690

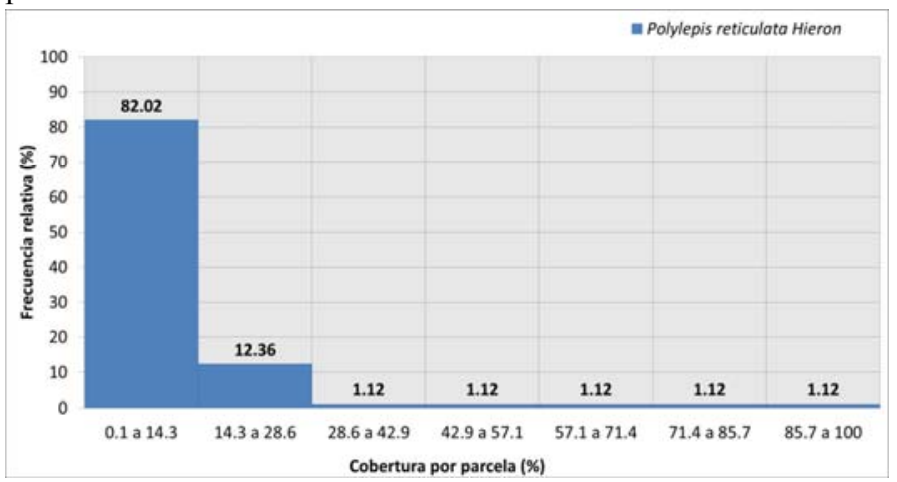

Figura 10. Distribución de coberturas de los renuevos de $P$. reticulata en sub-parcelas de $25 \mathrm{~m}^{2}$. msnm.

En líneas generales, se deduce que el bosque se encuentra en plena regeneración natural por la alta densidad poblacional, y por la presencia de regeneración en el $82 \%$ de las parcelas. Según diferentes autores, y de acuerdo a lo observado, estos individuos regenerados proceden de una reproducción generalmente vegetativa por rizomas subterráneos (Yallico, 1992) y en menor proporción por acodos naturales (Clavijo, 2012); aunque aún se desconoce si estos individuos pueden llegar a constituirse en árboles o por el contrario solamente ser matas de poco tamaño (Yallico, 1992). Polylepis presenta además reproducción por semilla pero con un bajo porcentaje de geminación, alrededor del $4 \%$ (Betancourt \& Rojas, 2011).

Por otro lado, el bosque se caracteriza por contener

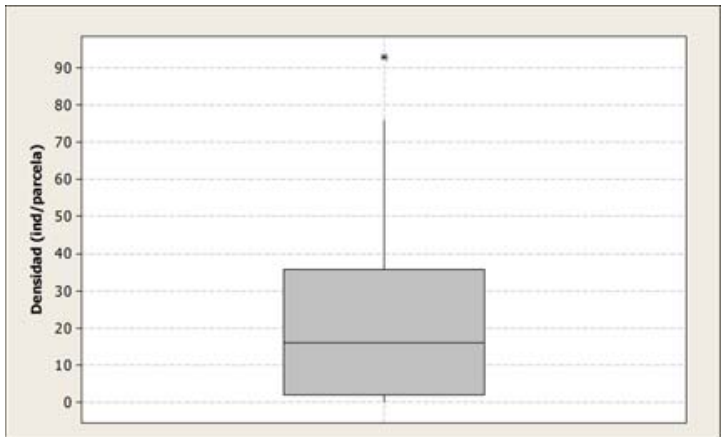

Figura 9. Distribución de densidades de los renuevos de $P$. reticulata en sub-parcelas de 25 $\mathrm{m}^{2}$.

renuevos bastante tiernos (alturas entre 8 a $33 \mathrm{~cm}$ ), esto podría explicarse porque el bosque se mantuvo en estado estacionario durante un largo periodo de tiempo y recién ha iniciado su proceso de regeneración. Otra hipótesis válida, es que la regeneración habría sido afectado por la presión de origen animal que pudo haber anteriormente, ya que comprimen la capa de musgo u hojarasca de la superficie del suelo, que fungen como "camas de germinación", volviendo vulnerables a los renuevos a condiciones climáticas adversas (Servat et al. 2013). La quema de pastos presente en algunos sectores del bosque, es también un factor que influenciaría en la distribución y crecimiento de los renuevos (Renison et al., 2002). Cabe resaltar que hay zonas bastante densas con cobertura arbórea superior a $80 \%$, que conllevaría a los individuos regenerados a no poder desarrollarse (no reciben luz solar) ni expandir sus raíces. 


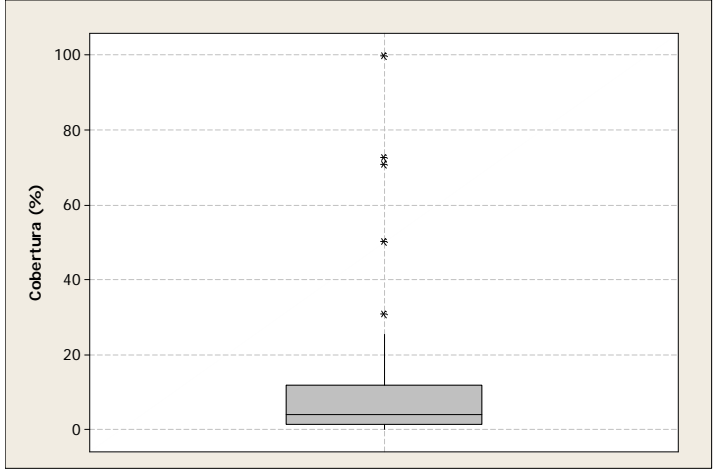

Figura 11. Distribución de coberturas de los renuevos de $P$. reticulata en sub-parcelas de $25 \mathrm{~m}^{2}$.

Con relación a los renuevos del bosque, se puede afirmar que por lo general son más desarrollados en la zona más alta del bosque donde se ubica $P$. sericea, a pesar de que en dicha zona no hay presencia de renuevos con alturas menores a $10 \mathrm{~cm}$. Esto se puede deberse al disturbio causado por la presión del ganado (Servat et al., 2013) que pastan cerca a los límites del bosque.

Se deduce que en general, el bosque de Huasta está constituido por individuos relativamente jóvenes (diámetros entre 9 a $26 \mathrm{~cm}$ ), en contraste con la menor cantidad de árboles semilleros (representa 1\%, diámetros entre 80 a $110 \mathrm{~cm}$ ), por lo que se puede inferir que puede tratarse de un bosque primario; ya

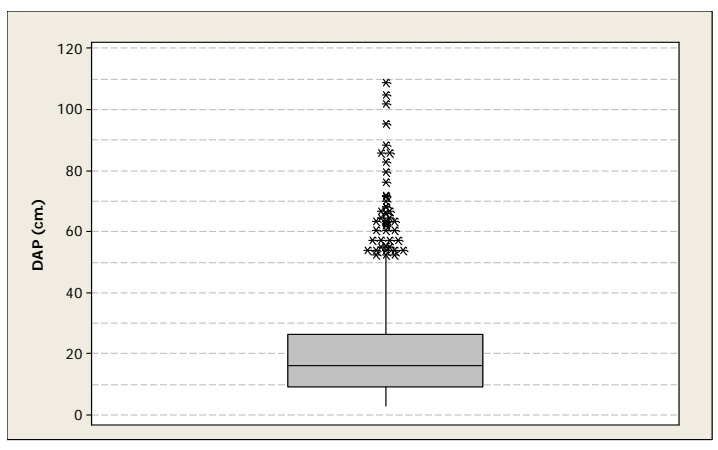

Figura 14. Distribución de diámetros $(\mathrm{cm})$ de los árboles de $P$. reticulata.

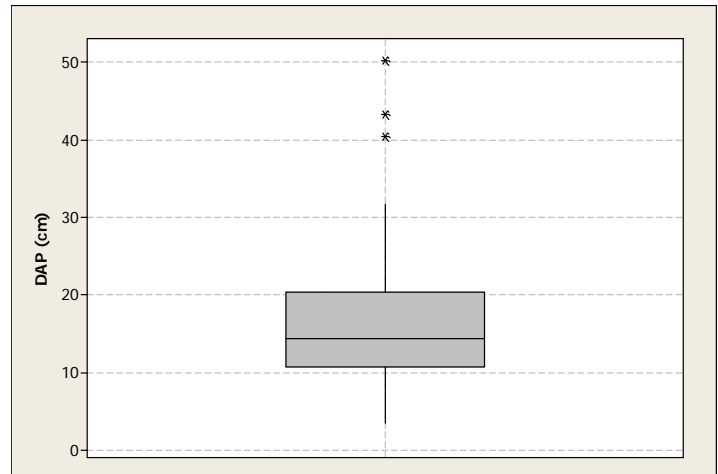

Figura 15. Distribución de diámetros $(\mathrm{cm})$ de los árboles de $P$. sericea.

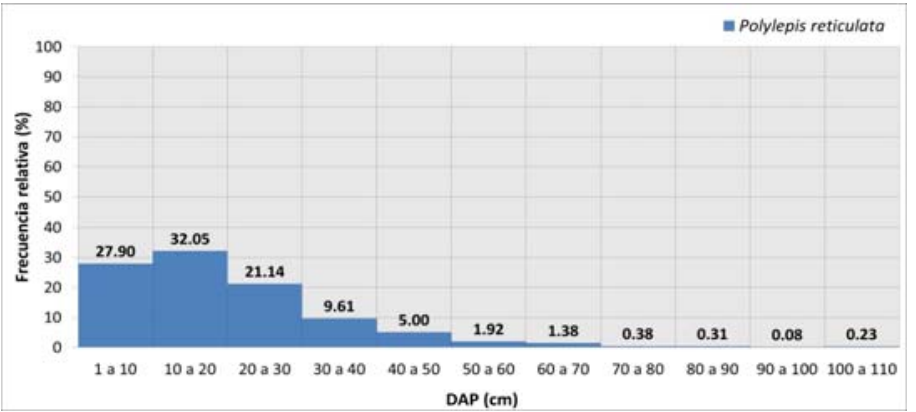

Figura 12. Distribución de diámetros de los árboles de P. reticulata.

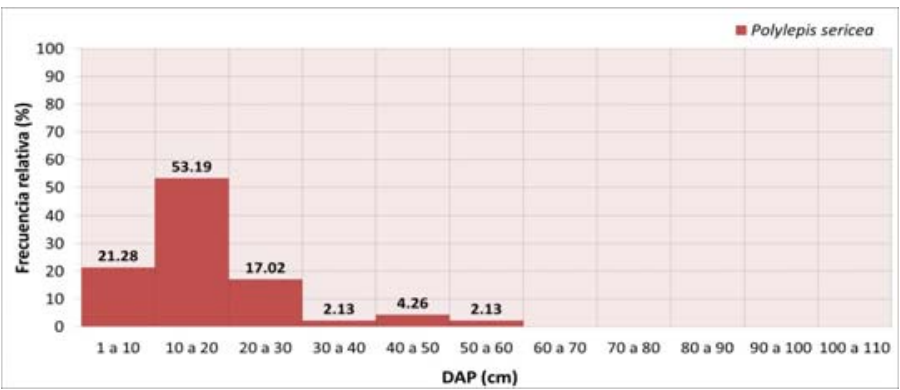

Figura 13. Distribución de diámetros de los árboles de $P$. sericea. que por su lento crecimiento (Betancourt \& Rojas, 2011) han debido de pasar cientos de años (Moya \& Lara, 2011) para que la capacidad de reproducción del bosque llegue a generar la gran cantidad de individuos jóvenes que se observan actualmente. Además, el bosque pudo haberse encontrado en estado de latencia durante un periodo largo de tiempo hasta que se iniciara la regeneración del mismo nuevamente, es por ello que, actualmente no hay presencia de individuos de porte arbustivo.

En comparación con investigaciones anteriores (Yallico, 1992; ECOAN, 2005; Kessler 2006; Flores et al., 2009; Servat et al., 2013), el bosque en estudio sería uno de los más antiguos del Perú, ya que hay presencia de árboles que sobrepasan los $90 \mathrm{~cm}$ de DAP, dato máximo encontrado en otros bosques del género. Además, el valor máximo encontrado (DAP $108.86 \mathrm{~cm}$ ) se constituye en el mayor registro reportado hasta la actualidad tanto para la especie $P$. reticulata, como para el género 


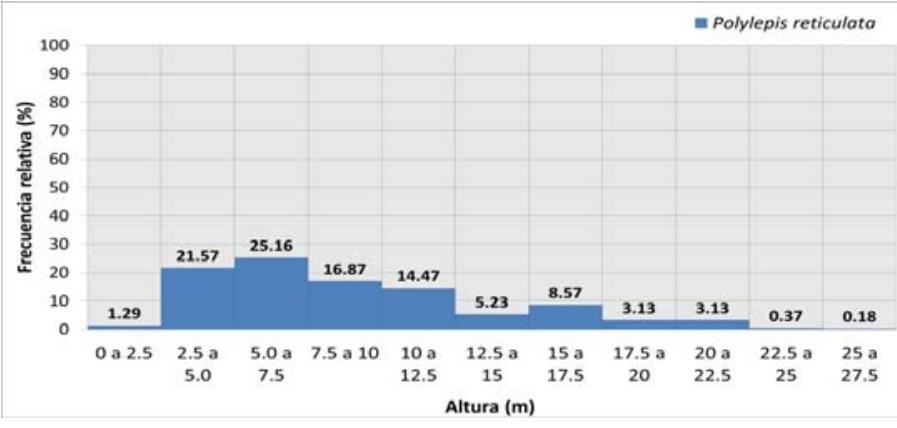

Figura 16. Distribución de alturas de los árboles de $P$. reticulata.

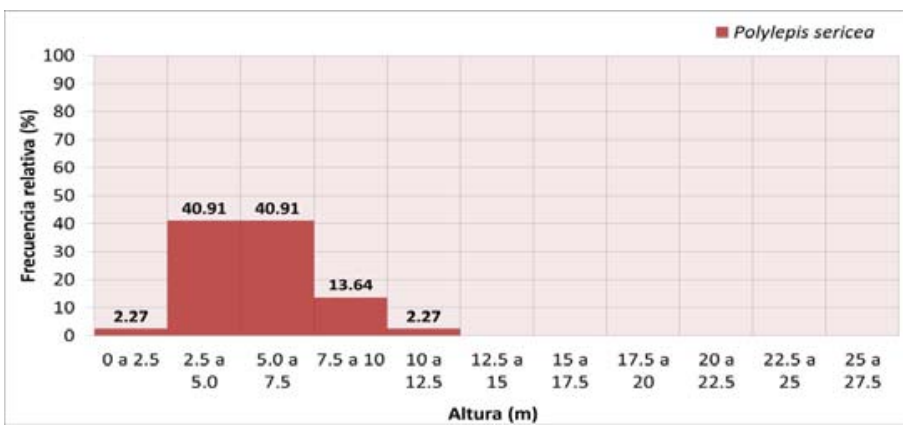

Figura 17. Distribución de alturas de los árboles de $P$. sericea.

Polylepis. Por otro lado, este bosque está constituido por individuos de mediana altura (5 a $11 \mathrm{~m})$, pero excepcionalmente se presentan árboles que pueden llegar a medir hasta los $26 \mathrm{~m}$, por lo que el bosque presentaría los árboles de Polylepis más altos del departamento de Ancash y del Perú, ya que se incluiría a la especie $P$. reticulata dentro del selecto grupo de especies del género que superarían los $25 \mathrm{~m}$ de altura al igual que las especies $P$. lanata y $P$. pauta (Kessler, 2006).

Por último el bosque en estudio presenta una alta densidad en comparación con estudios anteriores realizados a la misma especie. Caranqui (2011) encontró para $P$. reticulata una densidad de 217 indiv./ha, mientras que en el bosque en estudio se encontró una densidad de 605 indiv./ha.

\section{Conclusiones.}

El bosque de Huasta está conformado por dos especies, $P$. reticulata Hieron. que ocupa 18.5 ha y $P$. sericea Wedd. que ocupa 4.5 ha.

La especie $P$. reticulata se encuentra formando bosque hasta una altitud máxima de $4641 \mathrm{msnm}$, constituyéndose en la mayor altitud registrada hasta la fecha. Lo mismo sucede con la especie Polylepis sericea que se encuentra formando bosque hasta una altitud máxima de $4691 \mathrm{msnm}$.

Hay presencia de regeneración natural en el $82 \%$ de las parcelas a lo largo del bosque. La regeneración natural de Polylepis reticulata, se caracterizó por presentar alturas entre 8 a $33 \mathrm{~cm}$, densidades por lo general entre 11 a 42 individuos/parcela y una cobertura vegetal entre 1.3 a $11.7 \%$. Por otro lado, la regeneración natural de Polylepis sericea, se caracterizó por presentar alturas entre 25 a $63 \mathrm{~cm}$.

Los individuos de porte arbóreo de Polylepis reticulata presentaron por lo general DAP's entre 9.4 a $26.4 \mathrm{~cm}$, densidades entre 6 a 17 individuos/parcela y alturas entre 5 a $11.25 \mathrm{~m}$. En cambio, los árboles de Polylepis sericea (presente en $5 \%$ de las parcelas), presentaron DAP's entre 10.82 a $20.37 \mathrm{~cm}$ y alturas entre 4 a $7 \mathrm{~m}$.

Los árboles de Polylepis reticulata presentaron DAP's hasta un máximo de $108.86 \mathrm{~cm}$, constituyéndose en el mayor valor reportado para la especie y para el género. Por otro lado, Polylepis sericea presentó individuos de hasta $50 \mathrm{~cm}$ de DAP, constituyéndose en el mayor registro hasta ahora reportado para la especie.

Los árboles de Polylepis reticulata presentaron alturas hasta un máximo de $26 \mathrm{~m}$, constituyéndose en el mayor

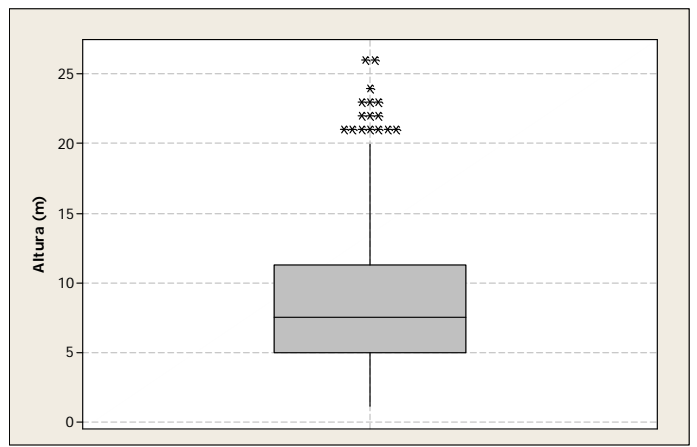

Figura 18. Distribución de alturas de los árboles de $P$. reticulata.

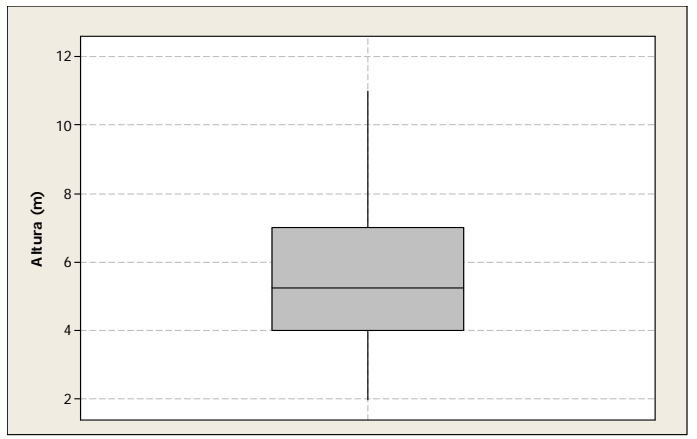

Figura 19. Distribución de alturas de los árboles de $P$. sericea. 
Enero - Julio 2015

registro reportado para la especie.

\section{Agradecimientos.}

A la ONG "The Mountain Institute" por financiar la realización del presente estudio. A la Lic. Est. Ana Vargas por el apoyo brindado en todo momento con sus conocimientos de estadística. A Cciary Alegría, Betzabeth Ávila, Anibal Loyola, Manilda Julca, Irving Guimel, Jose Julca y a Christian Julca por su apoyo durante el levantamiento de datos en el campo. A Carlos Sánchez y Andrea Castro por su apoyo en la revisión final del presente trabajo. Finalmente a Marlene Gonzales, Juan Castro, Ale Au, Andrea Castro y Jhudá Castro por su constante apoyo y compañía.

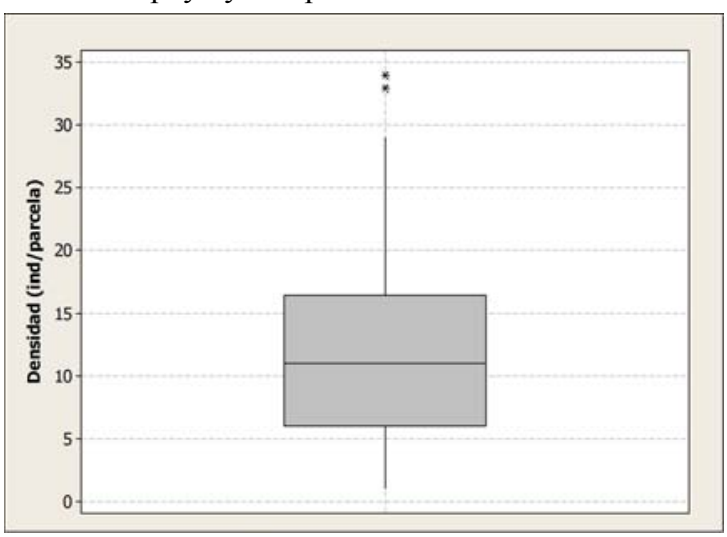

Figura 21. Distribución de densidades de los árboles de $P$. reticulata en sub-parcelas de $200 \mathrm{~m}^{2}$.

\section{Literatura citada.}

Arévalo R. \& Recharte J. 2003. Bosques de montaña: Ecosistemas relictos. In $\mathrm{J}$ Recharte, J; Arévalo, R; Glave M. eds. Islas del cielo. Ancash, PE. p. 11-19.

Betancourt J. \& Rojas M. 2011. Rizogénesis in vitro a partir de yemas de Polylepis incana Kunth., y Polylepis pauta Hieron., con la ulterior determinación de la especie viable para crecimiento in situ en la zona de Papallacta. Tesis (Ingeniero en Biotecnología de los Recursos Naturales). Universidad Politécnica Salesiana SedeQuito, EC.

Brack A., Mendiola C. \& Vargas M. 2000. Ecología del Perú. Editorial Bruño, Lima, PE. 495 pp.

Caranqui J. 2011. Demografía de un rodal de Polylepis reticulata Hieron. en la reserva de producción faunística Chimborazo. Herbario Escuela Superior Politécnica del Chimborazo-Escuela de Ingeniería Forestal. Chimborazo, EC.

Clavijo X. 2012. Monitoreo de poblaciones de plantas en bosques de Quínoa (Polylepis spp.) en el Parque Nacional Cajas. Universidad del Azua, Facultad de Ciencia y Tecnología, EC.

ECOAN (Asociación de Ecosistemas Andinos, PE). 2005. Evaluación de la biodiversidad de los bosques de
Polylepis del corredor de Conchucos - Huaraz. In Proyecto: Corredor de conservación de los bosques de Polylepis en el sur de los Conchucos. Ancash, PE.

Fjeldså J. \& Kessler M. 1996. Conserving the Biological Diversity of Polylepis Woodlands of the highlands of Perú and Bolivia: A contribution to sustainable natural resource management in the andes. NORDECO, Copenhagen, DK. 249p.

Flores C., Cruzado L., Panduro J., Valdez M., Peche F., Cusquipoma A., Valle E., Valle V. \& Egoavil M. 2009. Queñuales (Polylepis spp.) en la Concesión para Conservación Alto Huayabamba, San Martín: Primera aproximación. Amazónicos por la Amazonía - AMPA, Moyobamba, PE.

Kessler M. \& Schmidt-Lebuhn A. 2006. Taxonomical and distributional notes on Polylepis. Organisms Diversity \& Evolution 6

Kessler M. 2006. Bosques de Polylepis. Universidad Mayor de San Andrés, La Paz, BO.

León D. 2009. Propagación de dos Especies de Yagual (Polylepis incana y Polylepis racemosa) utilizando dos Enraizadores Orgánicos y dos Enraizadores Químicos en el Vivero Forestal del Crea en el Cantón y Provincia del Cañar. Tesis (Ingeniero Agrónomo). Riobamba, EC. Escuela Superior Politécnica de Chimborazo, Facultad de Recursos Naturales. $126 \mathrm{p}$

Mendoza W. \& Cano A. 2011. Diversidad del Género Polylepis (Rosaceae, Sanguisorbeae) en los Andes Peruanos. Rev. peru. biol. 18(2): 197 - 200. Lima, PE.

Minitab Inc. 2010. Software Minitab 16.1.0. Pennsylvania, US.

Moya J. \& Lara A. 2011. Cronologías de ancho de anillos de queñoa (Polylepis tarapacana) para los últimos 500 años en el Altiplano de la región de Arica y Parinacota, Chile. Bosque 32(2): 165-173. Arica, CH.

Renison D., Cingolani A. \& Suárez R. 2002. Efectos del fuego sobre un bosquecillo de Polylepis australis (Rosaceae) en las montañas de Córdova, Argentina. Revista Chilena de Historia Natural 75(4):719-727. Córdova, AR.

Rủgnitz M., Chacón M. \& Porro R. 2009. Guía para la Determinación de Carbono en Pequeñas Propiedades Rurales. Lima, PE, Centro Mundial de Agroforestal/Consorcio Iniciativa Amazónica. p. 13-24.

Servat G., Mendoza W. \& Ochoa A. 2002. Flora y fauna de cuatro bosques de Polylepis (Rosaceae) en la Cordillera 
del Vilcanota (Cusco, Perú). Ecología Aplicada, 1(1):2535. Universidad Agraria La Molina, Lima, PE.

Servat G., Mendoza W., Hurtado N., Castañeda R., Olarte M. \& Alcocer R. 2002. Crecimiento, regeneración y fenología de árboles de Polylepis pauta Hieron. (Rosaceae) en el ecotono del Bosque Montano del Valle del Río Apurímac. Monitoreo de Biodiversidad: Lecciones de un Megaproyecto Transandino, 1(1):245-
251. Editorial Smithsonian Institution Scholarly Press, Washington D.C., US.

Simpson B. 1979. A revision of the genus Polylepis. Smithsonian institution press. Washington, US.

Venero J. \& De Macedo H. 1983. Relictos de bosque en la Puna del Perú. Boletín de Lima 30:19 - 26.

Yallico E. 1992. Distribución de Polylepis en el sur de Puno. In Proyecto Árbol Andino. Puno, PE. 134 p.

${ }^{1}$ Facultad de Ciencias, Universidad Nacional Agraria La Molina, Av. La Molina S/N, Apartado Postal 15-024, Lima - Perú, eco.jacastro@gmail.com.

${ }^{2}$ Herbario MOL (A. Weberbauer), Departamento Académico de Biología, Universidad Nacional Agraria La Molina, Av. La Molina S/N, Apartado Postal 15-024, Lima - Perú, mflores@lamolina.edu.pe. 